\title{
Alantolactone Induces Apoptosis in HepG2 Cells through GSH Depletion, Inhibition of STAT3 Activation, and Mitochondrial Dysfunction
}

\author{
Muhammad Khan, ${ }^{1,2}$ Ting Li, ${ }^{1}$ Muhammad Khalil Ahmad Khan, ${ }^{2}$ Azhar Rasul, \\ Faisal Nawaz, ${ }^{3}$ Meiyan Sun, ${ }^{1}$ Yongchen Zheng, ${ }^{1}$ and Tonghui $\mathrm{Ma}^{1}$ \\ ${ }^{1}$ Central Research Laboratory, Jilin University Bethune Second Hospital, Changchun 130041, China \\ ${ }^{2}$ Department of Zoology, University of the Punjab, Quaid-e-Azam Campus, Lahore 54590, Pakistan \\ ${ }^{3}$ Key Laboratory of Inorganic Synthesis and Preparative Chemistry, Jilin University, Qianjin Street, Changchun 130012, China \\ Correspondence should be addressed to Yongchen Zheng; jlzyc@yahoo.com.cn
}

Received 2 August 2012; Revised 24 October 2012; Accepted 24 October 2012

Academic Editor: D. M. Clarke

Copyright ( 2013 Muhammad Khan et al. This is an open access article distributed under the Creative Commons Attribution License, which permits unrestricted use, distribution, and reproduction in any medium, provided the original work is properly cited.

\begin{abstract}
Signal transducer and activator of transcription 3 (STAT3) constitutively expresses in human liver cancer cells and has been implicated in apoptosis resistance and tumorigenesis. Alantolactone, a sesquiterpene lactone, has been shown to possess anticancer activities in various cancer cell lines. In our previous report, we showed that alantolactone induced apoptosis in U87 glioblastoma cells via GSH depletion and ROS generation. However, the molecular mechanism of GSH depletion remained unexplored. The present study was conducted to envisage the molecular mechanism of alantolactone-induced apoptosis in HepG2 cells by focusing on the molecular mechanism of GSH depletion and its effect on STAT3 activation. We found that alantolactone induced apoptosis in HepG2 cells in a dose-dependent manner. This alantolactone-induced apoptosis was found to be associated with GSH depletion, inhibition of STAT3 activation, ROS generation, mitochondrial transmembrane potential dissipation, and increased Bax/Bcl-2 ratio and caspase-3 activation. This alantolactone-induced apoptosis and GSH depletion were effectively inhibited or abrogated by a thiol antioxidant, N-acetyl-L-cysteine (NAC). The data demonstrate clearly that intracellular GSH plays a central role in alantolactoneinduced apoptosis in HepG2 cells. Thus, alantolactone may become a lead chemotherapeutic candidate for the treatment of liver cancer.
\end{abstract}

\section{Introduction}

Hepatocellular carcinoma is currently the fifth most common cancer and third leading cause of cancer-related deaths in the world. Over 600000 patients die because of liver cancer in the world every year. Despite significant advances in surgery and chemotherapy, the majority of patients with hepatocellular carcinoma die within one year of diagnosis [1-4]. At present, the hepatocellular carcinoma is mainly treated with surgery and chemotherapy [5,6]. Currently, doxorubicin is the most widely used drug against liver cancer either as single agent or in combination with other chemotherapeutics like cisplatin. However, the outcomes of the existing conventional chemotherapeutic drugs remain considerably low due to their severe toxicity on normal hepatocytes $[7,8]$. Therefore, searching for highly efficient anticancer drugs with low hepatotoxicity remains a hot research area.

A causal link between chronic inflammation and development of cancer is well established. Many transcription factors such as NF- $\kappa$ B and STAT3 are key to innate inflammation. The constitutive activation of signal transducers and activators of transcription 3 (STAT3) has been frequently detected in many types of human cancers such as glioblastoma, myeloma, colorectal, and hepatocellular carcinoma where it plays an important role in cell proliferation, tumor invasion, metastasis, and drug resistance $[9,10]$. Therefore, blockage of STAT3 may have a therapeutic potential in preventing and treating these cancers. 


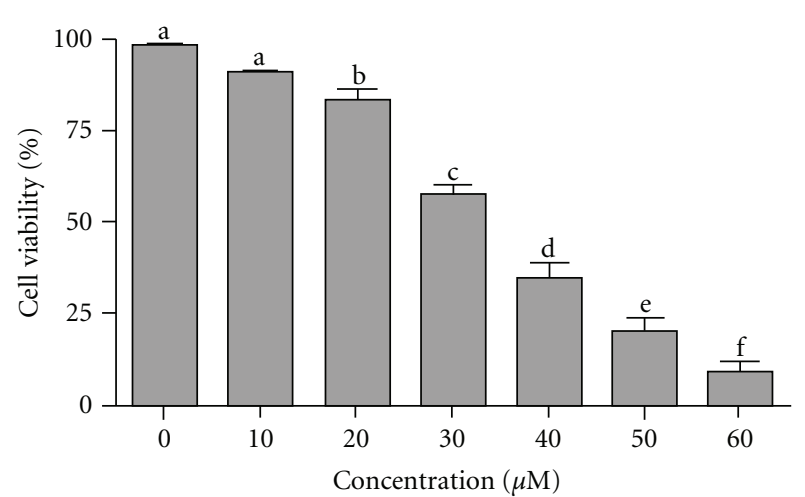

FIGURE 1: Growth inhibition of HepG2 cells after the treatment with alantolactone. Cells were cultured for $24 \mathrm{~h}$ before drug treatment in 96 well plates. Cells were treated with alantolactone $(0,10,20,30$, 40,50 , and $60 \mu \mathrm{M}$ ) for $12 \mathrm{~h}$ and cell viability was measured by MTT assay. Data are expressed as mean $\pm \mathrm{SD}(n=3)$. Columns not sharing the same superscript letter differ significantly $(P<0.05)$.

Sesquiterpene lactones are plant-derived bioactive constituents often used in traditional medicines against inflammation and cancer $[11,12]$. Alantolactone, a sesquiterpene lactone component of Inula helenium and Inula racemosa, has been shown to exhibit multiple pharmacological activities including anticancer effect $[13,14]$. In our previous report, we have shown that alantolactone induces apoptosis in U87 glioblastoma cells via GSH depletion and mitochondrial dysfunction. However, the molecular mechanism of GSH depletion by alantolactone remained largely unknown. Furthermore, we showed that alantolactone did not induce hepatotoxicity and nephrotoxicity in mice [15]. Additionally, Butturini et al. showed that GSH depletion is involved in the inhibition of STAT3 activation [16].

Keeping in mind the antiinflammatory effect and GSH depleting activity of sesquiterpene lactones, we hypothesized that alantolactone can inhibit STAT3 activation and induce apoptosis in HepG2 cells. To evaluate this, we investigated the effect of alantolactone on GSH depletion and STAT3 and its downstream target gene Bcl-2 expressions. The data demonstrated that alantolactone-induced apoptosis in HepG2 cells via GSH depletion, STAT3 inhibition, modulation of Bcl-2 family proteins, and caspase- 3 activation.

\section{Materials and Methods}

Alantolactone was obtained from Tauto Biotech Co., Ltd., (Shanghai, China) and purity (>99\%) was determined by HPLC (see Supplementary material available online at doi:10.1155/2013/719858, Figure 1). Propidium iodide (PI) calcein acetoxymethyl ester (Calcein AM), Rhodamine 123, Dimethyl Sulfoxide (DMSO), MTT, Dulbecco's Modified Eagle's Medium (DMEM), fatal bovine serum (FBS), penicillin, and streptomycin were purchased from Sigma (Beijing, China). Apoptosis assay kit was purchased from KeyGen (Shanghai, China) while reactive oxygen species kit, and GSH/GSSG assay kit were purchased from Beyotime Institute of Biotechnology (Haimen, Jiangsu, China).
Antibodies specific to $\mathrm{Bax}, \mathrm{Bcl}-2$, caspase- 3 , and $\beta$-actin were obtained from Beyotime while $\mathrm{pTyr}^{705}$ STAT3 was obtained from Wuhan Boster Biological Technolgy, Ltd., (Wuhan, China). Antibody specific to glutathione reductase was obtained from BIOSS Beijing Biosynthesis Biotechnology, Co., ltd. Horseradish peroxidase-conjugated secondary antibodies (goat-antirabbit, goat-antimouse) were purchased from Sigma Aldrich.

2.1. Cell Culture and Treatment. The human HepG2 cells were obtained from Shanghai Cell Bank, China, and were cultured in Dulbecco's Modified Eagle's Medium (DMEM) supplemented with $10 \%$ fatal bovine serum (FBS), 100 units $/ \mathrm{mL}$ penicillin and $100 \mu \mathrm{g} / \mathrm{mL}$ streptomycin and maintained at $37^{\circ} \mathrm{C}$ with $5 \% \mathrm{CO}_{2}$ in humidified atmosphere. Cells were treated with alantolactone dissolved in DMSO with a final DMSO concentration of $1 \%$. DMSO treated cells were used as control in all the experiments.

2.2. Determination of Cell Viability. Cell viability was determined by MTT assay as described by us previously [17]. Briefly HepG2 cells were treated with different concentrations of alantolactone for $12 \mathrm{~h}$. Following treatment, the MTT reagent was added $(500 \mu \mathrm{g} / \mathrm{mL})$ and cells were further incubated at $37^{\circ} \mathrm{C}$ for $4 \mathrm{~h}$. Subsequently $150 \mu \mathrm{L}$ DMSO was added to dissolve farmazan crystals and absorbance was measured at $570 \mathrm{~nm}$ in a microplate reader (Thermo Scientific). The percentage of cell viability was calculated as follows:

$$
\text { Cell viability }(\%)=\frac{\left(A 570_{\text {sample }}-A 570_{\text {blank }}\right)}{\left(A 570_{\text {control }}-A 570_{\text {blank }}\right)} \times 100 \text {. }
$$

The $\mathrm{IC}_{50}$ values were calculated using GraphPad Prism 5.

2.3. Observation of Morphological Changes. HepG2 cells were treated with $40 \mu \mathrm{M}$ alantolactone in the presence or absence of NAC for $0,3,6$, and $12 \mathrm{~h}$. Cell morphological changes were observed by phase contrast microscopy (Olympus 1x71).

2.4. Live/Dead Assay. HepG2 cells were treated with $40 \mu \mathrm{M}$ alantolactone in the presence or absence of NAC for 0 , 3,6 , and $12 \mathrm{~h}$. Live and dead cells were quantified using fluorescent probe calcein AM and PI and fluorescence microscope as described by us previously [18]. Calcein AM is cell membrane permeable and stains only viable cells whereas PI is cell membrane impermeable and stains only dead cells. Treated and untreated cells were collected, washed with phosphate buffered saline (PBS), and incubated with PBS solution containing $2 \mu \mathrm{M}$ calcein AM and $4 \mu \mathrm{M}$ PI in the dark for $20 \mathrm{~min}$ at room temperature. Finally, 100 cells were counted microscopically for the percentage of live and dead cells.

2.5. Apoptosis Assay by Annexin V-FITC and Propidium Iodide (PI) Staining. HepG2 cells were treated with $40 \mu \mathrm{M}$ alantolactone for $0,3,6$, and $12 \mathrm{~h}$. After treatment, cells were harvested, washed with PBS, and resuspended in $500 \mu \mathrm{L}$ of binding buffer containing $5 \mu \mathrm{L}$ Annexin $\mathrm{V}$ and $5 \mu \mathrm{L} \mathrm{PI}$ and 


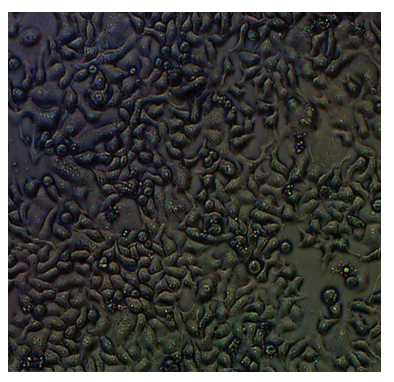

(a)

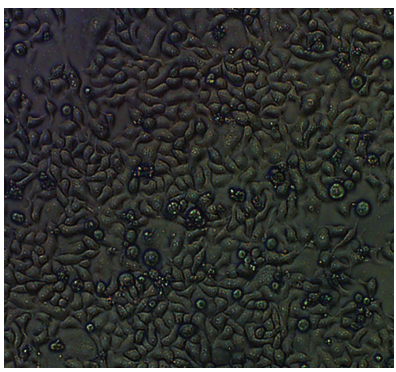

(e)

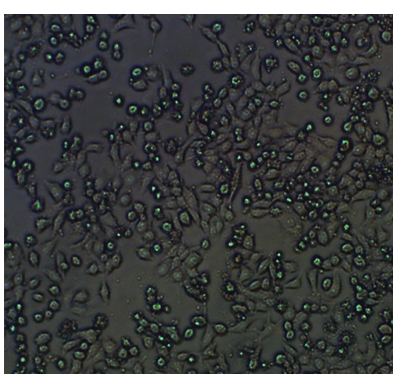

(b)

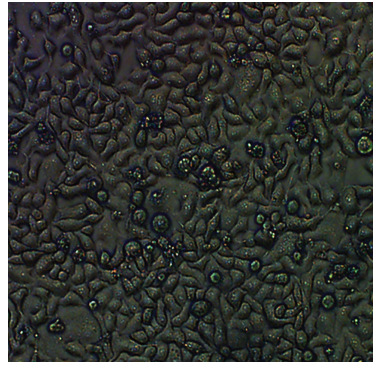

(f)

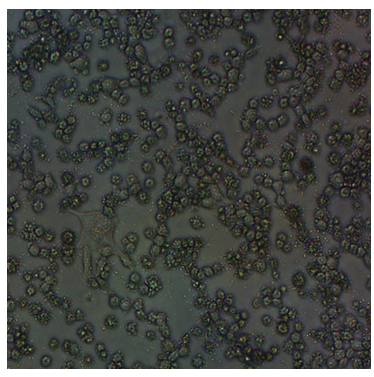

(c)

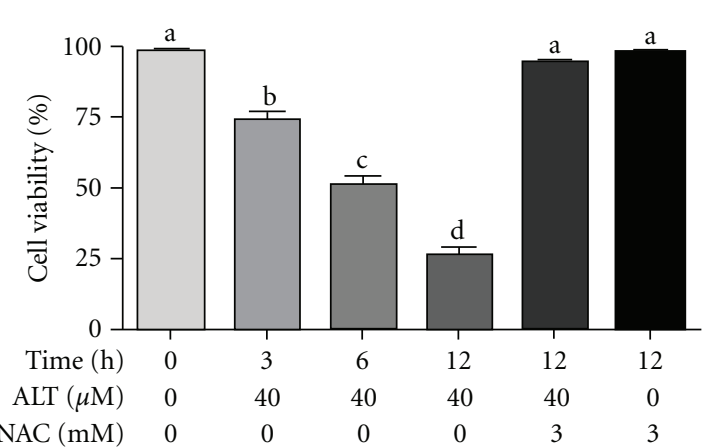

(g)

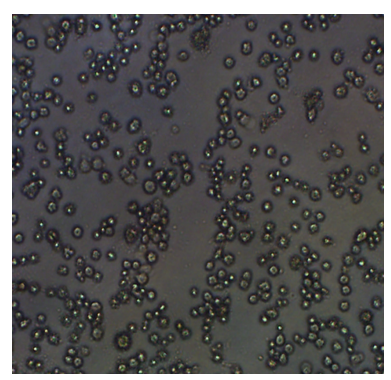

(d)

Figure 2: Changes in HepG2 cell morphology during alantolactone-induced cell death. HepG2 cells were treated with $40 \mu \mathrm{M}$ alantolactone in the presence or absence of $3 \mathrm{mM} \mathrm{NAC}$ for various time intervals and morphological changes were observed by phase contrast microscopy. (a) control, ((b), (c) and (d)) cells were treated with $40 \mu \mathrm{M}$ alantolactone for 3, 6, and $12 \mathrm{~h}$, (e) cells were treated with $40 \mu \mathrm{M}$ alantolactone in the presence of $3 \mathrm{mM} \mathrm{NAC}$ for $12 \mathrm{~h}$, and (f) cells were treated with NAC alone for $12 \mathrm{~h}$, respectively. (g) Cells were treated with $40 \mu \mathrm{M}$ alantolactone as described above and live and dead cells were quantified using fluorescent probe calcein AM and PI as described in Section 2. Data are expressed as mean $\pm \mathrm{SD}(n=3)$. Columns not sharing the same superscript letter differ significantly $(P<0.05)$.

put in the dark for 15 min according to the kit instructions (KeyGen, Shanghai, China). After incubation, samples were immediately analyzed by flow cytometry (Beckman Coulter, Epics XL).

2.6. Measurement of Reactive Oxygen Species (ROS). The intracellular changes in ROS generation were measured by staining the cells with $2^{\prime}, 7^{\prime}$-dichlorofluorescein-diacetate (DCFH-DA) as described previously [18]. The fluorescent dye DCFH-DA is a cell membrane permeable and is converted into cell membrane impermeable nonfluorescent compound DCFH by intracellular esterases. Oxidation of DCFH by reactive oxygen species produces a highly fluorescent DCF. The fluorescence intensity of DCF inside the cells is proportional to the amount of peroxide produced. Briefly HepG2 cells were treated with $40 \mu \mathrm{M}$ alantolactone for 0,3 , 6 , and $12 \mathrm{~h}$. After treatment, cells were further incubated with $10 \mu \mathrm{mol} / \mathrm{L} \mathrm{DCFH}-\mathrm{DA}$ at $37^{\circ} \mathrm{C}$ for $30 \mathrm{~min}$. Subsequently cells were harvested, rinsed, resuspended in PBS, filtered with 300 apertures, and analyzed for $2^{\prime}, 7^{\prime}$-dichlorofluorescein (DCF) fluorescence by flow cytometry.

2.7. Measurement of Mitochondrial Membrane Potential $(M M P)$. Rhodamine 123 was used to evaluate the changes in mitochondrial membrane potential as described previously
[18]. Briefly HepG2 cells were incubated with $40 \mu \mathrm{M}$ Alantolactone for $0,3,6$, and $12 \mathrm{~h}$. Following incubation, cells were collected, resuspended in $1 \mathrm{~mL}$ PBS solution containing $10 \mu \mathrm{g}$ Rhodamine 123, and incubated in the dark for $30 \mathrm{~min}$. After incubation, cells were centrifuged at $500 \times \mathrm{g}$ for $5 \mathrm{~min}$, supernatant was removed, and the pellet was gently rinsed with PBS once and then resuspended in $500 \mu \mathrm{L}$ PBS. After filtration (300 apertures), the suspension was analyzed by flow cytometry.

2.8. Measurement of GSH and GSSG. The intracellular reduced (GSH) and oxidized (GSSG) glutathione was determined spectrophotometrically using GSH and GSSG assay kit (Beyotime). Briefly, HepG2 cells were treated with $40 \mu \mathrm{M}$ alantolactone for $0,3,6$, and $12 \mathrm{~h}$ or with $40 \mu \mathrm{M}$ alantolactone in the presence or absence of $2 \mathrm{mM}$ methionine and $3 \mathrm{mM}$ NAC for $6 \mathrm{~h}$. Following treatment, the intracellular and extracellular (medium) GSH and GSSG were measured according to the instruction of kit. The values were expressed as nmols $\mathrm{GSH} / \mathrm{mg}$ protein.

2.9. HPLC Analysis. One $\mathrm{mM}$ alantolactone was incubated with $0,5,15$, and $30 \mathrm{mM}$ GSH in DMEM medium without FBS at $37^{\circ} \mathrm{C}$ for $30 \mathrm{~min}$. Following incubation, the samples were analyzed by HPLC (Waters) using XTerra MS C18 


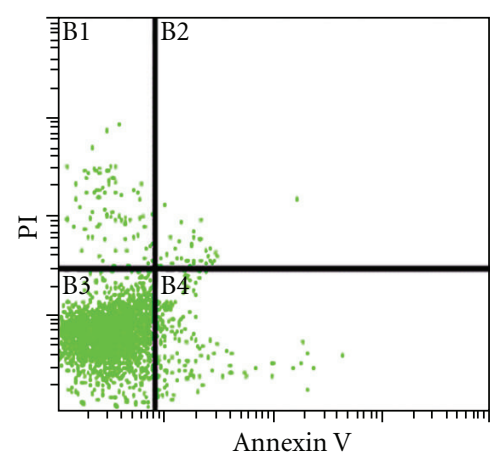

(a) $\mathrm{a}=$ control

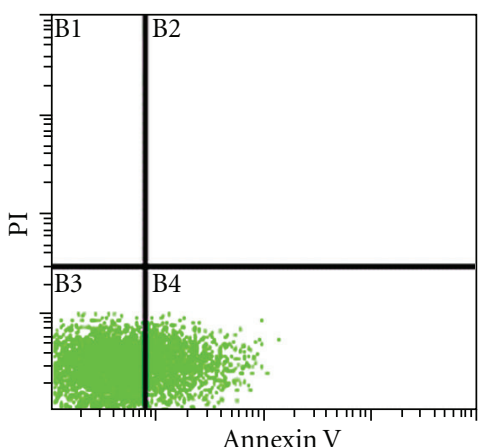

(b) $\mathrm{b}=3 \mathrm{~h}$

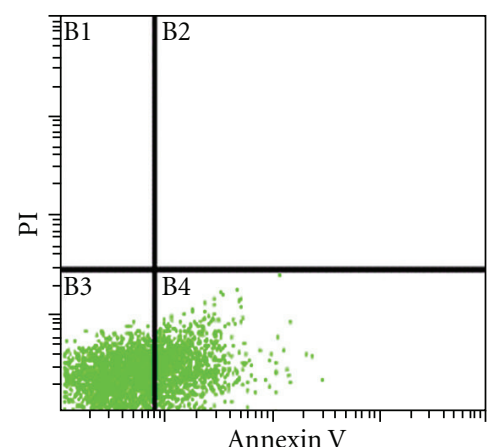

(c) $c=6 \mathrm{~h}$

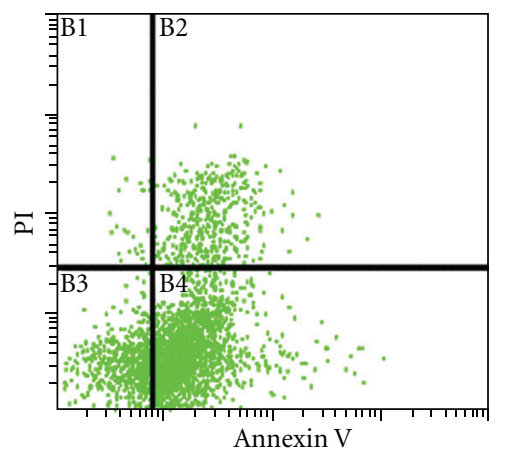

(d) $\mathrm{d}=12 \mathrm{~h}$

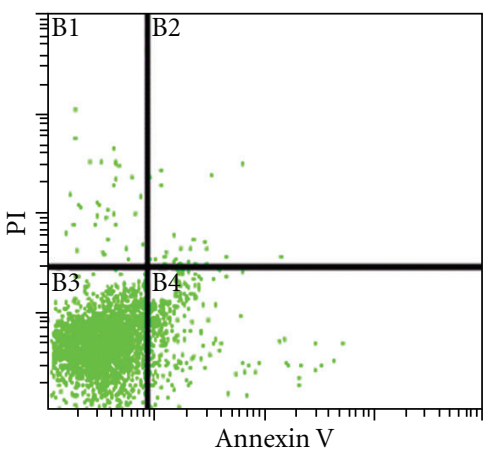

(e) $\mathrm{e}=12 \mathrm{~h}+\mathrm{NAC}$

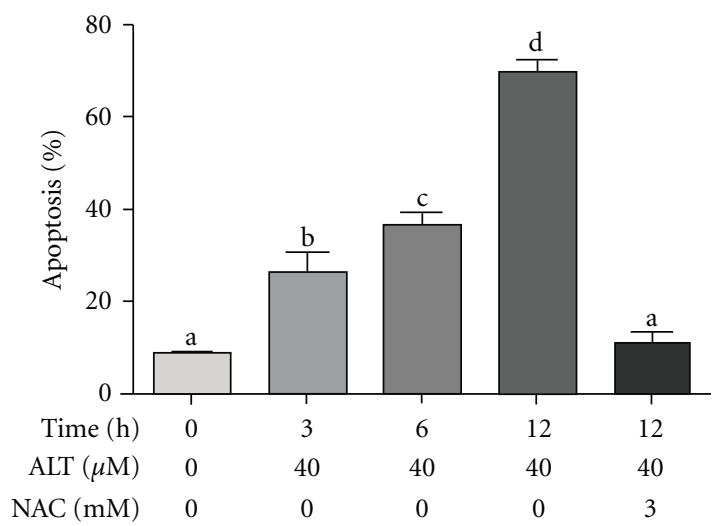

(f)

FIgURE 3: Flow cytometry analysis of apoptosis in HepG2 cells treated with $40 \mu \mathrm{M}$ alantolactone for various time points. (a) Control, ((b), (c) and (d)) cells were treated with $40 \mu \mathrm{M}$ alantolactone for 3,6, and $12 \mathrm{~h}$, respectively. (e) cells were treated with $40 \mu \mathrm{M}$ alantolactone in the presence of $3 \mathrm{mM} \mathrm{NAC}$ for $12 \mathrm{~h}$. (f) Data are expressed as mean $\pm \mathrm{SD}(n=3)$. Columns not sharing the same superscript letter differ significantly $(P<0.05)$.

$(5 \mu \mathrm{m}, 4.6 \times 150 \mathrm{~mm})$ column. The mobile phase was composed of acetonitrile $(\mathrm{A})$ and water $(\mathrm{B})$. The gradient program was as follows: $0-30 \mathrm{~min}, \mathrm{~A}=65 \%$, and $\mathrm{B}=35 \%$. The elution profile was analyzed at $227 \mathrm{~nm}$ by UV detector.

2.10. RNA Isolation and Semiquantitative RT-PCR. Total RNA was isolated from treated and untreated HepG2 cells using AxyPrep Multisource Total RNA Miniprep kit. cDNA was reverse-transcribed from $500 \mathrm{ng}$ of total RNA in a final volume of $10 \mu \mathrm{L}$ using PrimeScript RT reagent Kit (TakaRa, DRR037A), in accordance with the manufacturer's instructions. This was followed by 35 cycles of $94^{\circ} \mathrm{C}$ : $1 \mathrm{~min}$; $52^{\circ} \mathrm{C}$ : $30 \mathrm{sec} ; 72^{\circ} \mathrm{C}: 1 \mathrm{~min}$ ) and a final extension of $72^{\circ} \mathrm{C}$ for $10 \mathrm{~min}$. PCR product was visualized on $1 \%$ agarose gel containing ethidium bromide. The primers used were as follows:

$$
\gamma \text {-GCS, } 5 \text {-GGCACAGGTAAAACCAAATAGTAAC- } 3^{\prime}
$$
(Forward) and 5 -CAAATTG-TTTAGCAAATGCAGTCA$3^{\prime}$ (Reverse); GAPDH, 5'-ATGACATCAAGAAGGTGGTG-3' (Forward) and $5{ }^{\prime}$-CATACCAGGAAATGAGCTTG-3' (Reverse).

2.11. Immunoblotting. Proteins were isolated from control and alantolactone-treated cells as described previously [18].
$40 \mu \mathrm{g}$ proteins were electrophoresed on $12 \%$ SDS-PAGE and transferred to PVDF membrane. After blocking with $5 \%(\mathrm{w} / \mathrm{v})$ nonfat milk and washing with Tris-buffered salineTween solution (TBST), membranes were incubated for $2 \mathrm{~h}$ at room temperature with $\mathrm{Bax}(1: 300), \mathrm{Bcl}-2(1: 1000)$, Caspase-3, (1:500), pTyr ${ }^{705}$ STAT3 $(1: 300)$, Glutathione Reductase (GR) $(1: 300)$, and $\beta$-actin $(1: 400)$ antibodies, respectively. After washing, the blots were incubated with horse radish peroxidase conjugated goat antirabbit IgG or goat antimouse IgG secondary antibodies $(1: 5000)$ for $1 \mathrm{~h}$ at room temperature. After washing with TBST, signals were detected using ECL plus chemiluminescence kit (Millipore Corporation) on X-ray film.

2.12. Statistical Analysis. The results are expressed as mean \pm SD and statistically compared with control group or within the groups using one-way ANOVA followed by Tukey's multiple comparison test.

\section{Results}

3.1. Alantolactone Inhibits Growth of HepG2 Cells In Vitro. The effect of alantolactone on the growth of HepG2 cells was evaluated by MTT assay. Treatment with alantolactone 


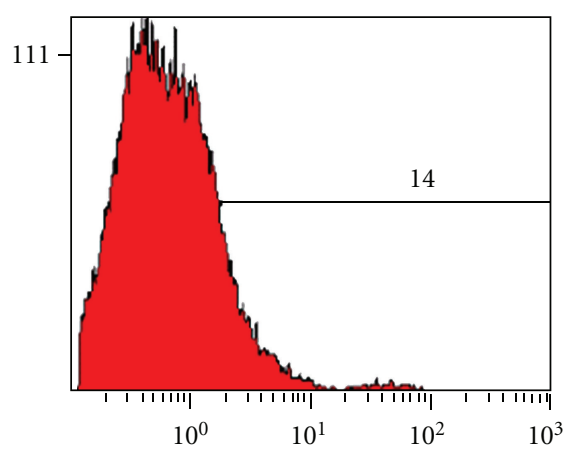

(a)

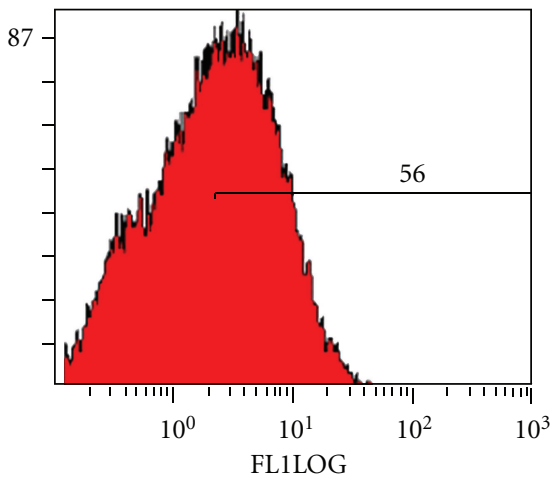

(d)

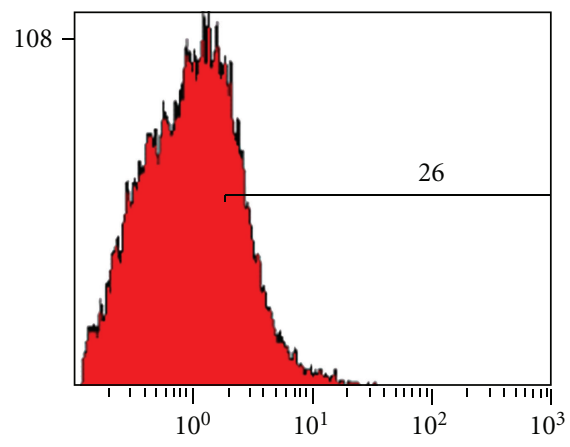

(b)

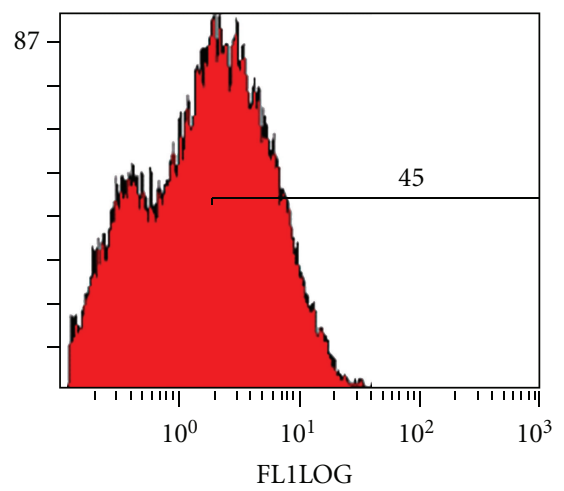

(c)

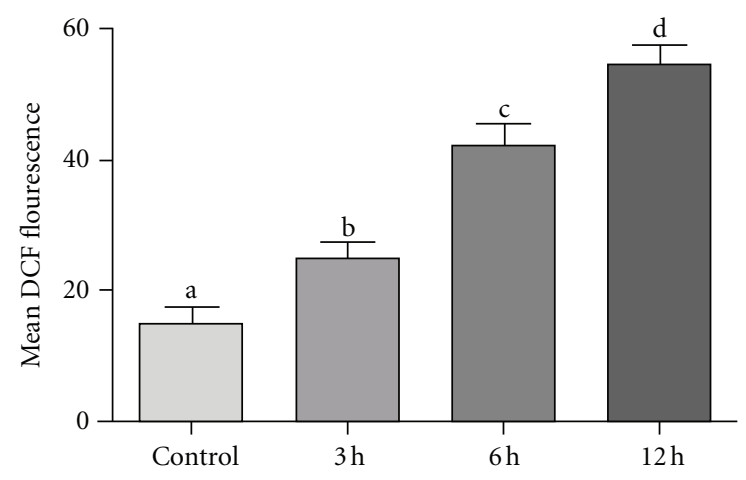

(e)

FIGURE 4: Flow cytometry analysis of ROS generation in control and alantolactone-treated HepG2 cells. (a) Control, ((b), (c) and (d)) cells were treated with $40 \mu \mathrm{M}$ alantolactone for 3,6 , and $12 \mathrm{~h}$, respectively. After treatment, cells were incubated with DCFH-DA for 30 min at $37^{\circ} \mathrm{C}$, washed with PBS, and analyzed for DCF fluorescence by flow cytometry. (e) Data are expressed as mean \pm SD $(n=3)$. Columns not sharing the same superscript letter differ significantly $(P<0.05)$.

for $12 \mathrm{~h}$ inhibited the growth of cells in a dose-dependent manner (Figure 1). The $\mathrm{IC}_{50}$ value of alantolactone was $33 \mu \mathrm{M}$ after $12 \mathrm{~h}$ treatment. $40 \mu \mathrm{M}$ concentration was selected for the following experiments.

3.2. Microscopic Study of HepG2 Cells. To examine the effect of alantolactone on cell morphology, HepG2 cells were treated with $40 \mu \mathrm{M}$ alantolactone for various time intervals $(3,6$, and $12 \mathrm{~h}$ ) and morphological changes were observed by phase contrast microscopy. The data showed that alantolactone-induced severe morphological changes of cell death including rounding and shrinkage of cells, in a timedependent manner (Figure 2). Pretreatment of cells with 3 mM NAC, a specific ROS inhibitor, completely protected the cells from cytotoxic effect of alantolactone. Furthermore, live and dead cells were quantified using fluorescent probes calcein AM and PI. As shown in Figure 2(g), alantolactone treatment reduced the viability of cells in a time-dependent manner. The viability of cells treated with $40 \mu \mathrm{M}$ alantolactone for 3,6 , and $12 \mathrm{~h}$ was $74.33 \%, 51.6 \%$, and $27 \%$, respectively. These values were significantly lower than those of the control group $(98.5 \%, P<0.05)$. Pretreatment of cells with $3 \mathrm{mM}$ NAC reversed the cytotoxic effect of alantolactone indicating that alantolactone exerts cytotoxic effect through the generation of ROS. However, NAC alone at this concentration did not affect the viability of cells as shown in Figure 2(g).

3.3. Alantolactone Induces Apoptosis in HepG2 Cells. The effect of alantolactone on cell apoptosis was evaluated by using annexin V-FITC/PI staining and flow cytometry. Translocation of phosphatidylserine (PS) to the outer leaflet of cellular membrane is the key step in the early stages of apoptosis. Annexin V selectively binds to PS and helps to identify cells undergoing apoptosis. When cells are double stained with Annexin V/PI, three different populations of cells can be observed. The cells that do not stain with either annexin $\mathrm{V}$ or PI are alive and reside in region B3; the cells that stain with only annexin $\mathrm{V}$ are in the stage of early apoptosis and reside in region B4 while the cells that stain with both reagents are nonviable late apoptotic/necrotic cells and scatter in region $\mathrm{B} 2$.

Flow cytometric analysis of apoptosis showed that alantolactone-induced apoptosis in HepG2 cells in a timedependent manner as shown in Figure 3. Moreover, the early and late apoptosis process was also time dependent. Treatment with $40 \mu \mathrm{M}$ alantolactone for 3 and $6 \mathrm{~h}$ only increased the early apoptosis while at $12 \mathrm{~h}$ late apoptosis 


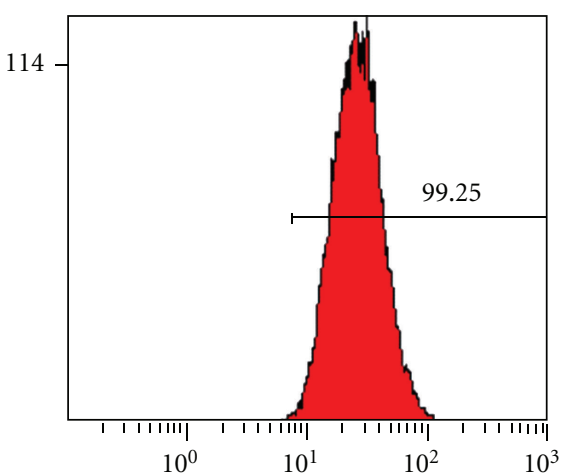

(a)

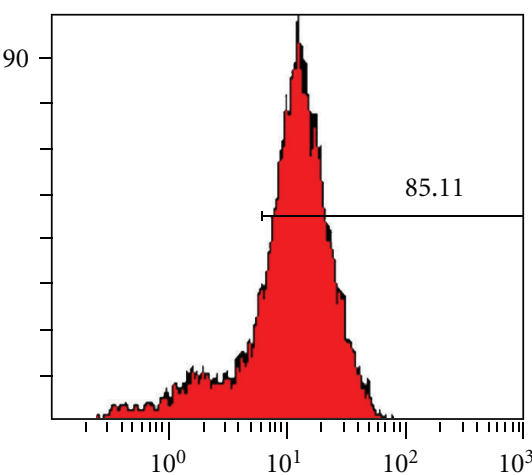

(b)

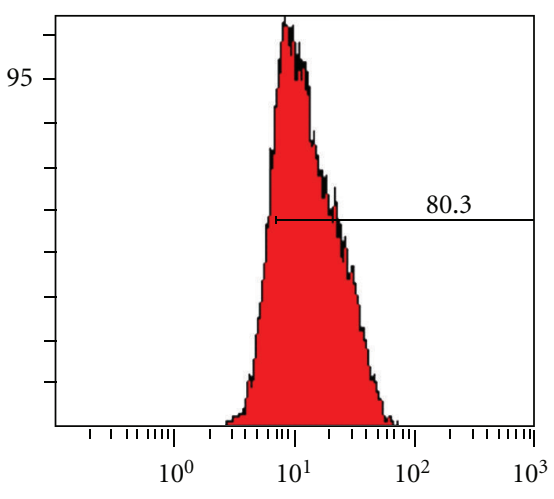

FL1LOG

(c)

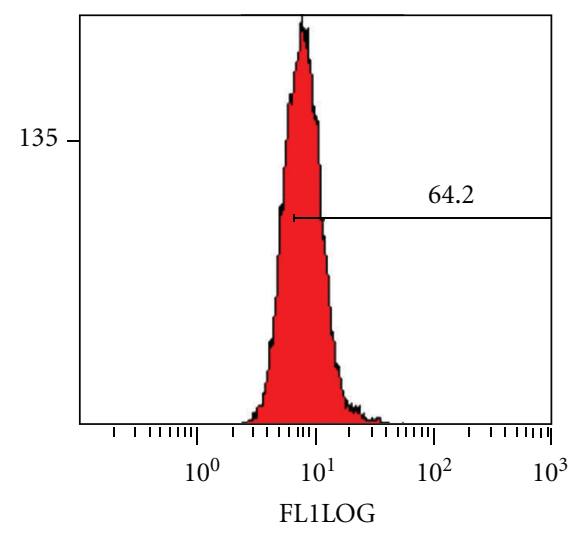

(d)

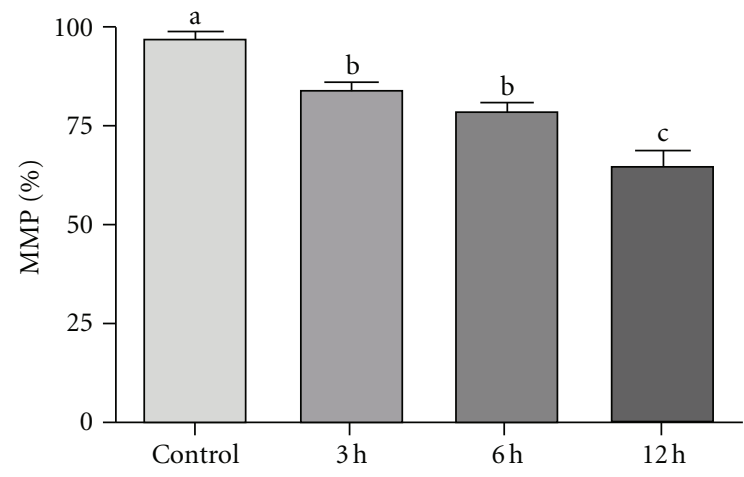

(e)

Figure 5: Flow cytometry analysis of MMP in control and alantolactone-treated HepG2 cells. (a) Control, (b), (c) and (d) cells were treated with $40 \mu \mathrm{M}$ alantolactone for 3, 6, and $12 \mathrm{~h}$, respectively. After treatment, cells were incubated with Rhodamine 123 for 30 min in dark, washed with PBS, and analyzed for MMP by flow cytometry. (e) Data are expressed as mean $\pm \mathrm{SD}(n=3)$. Columns not sharing the same superscript letter differ significantly $(P<0.05)$.

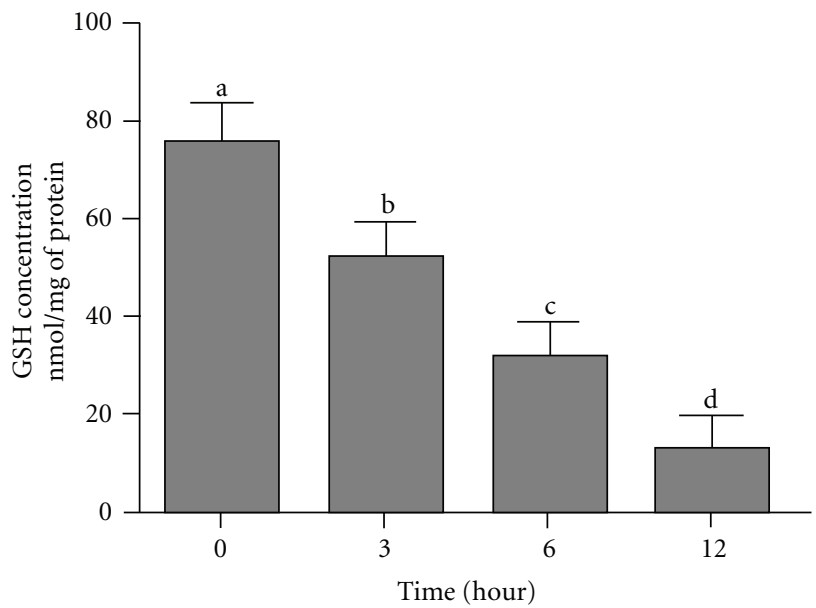

(a)

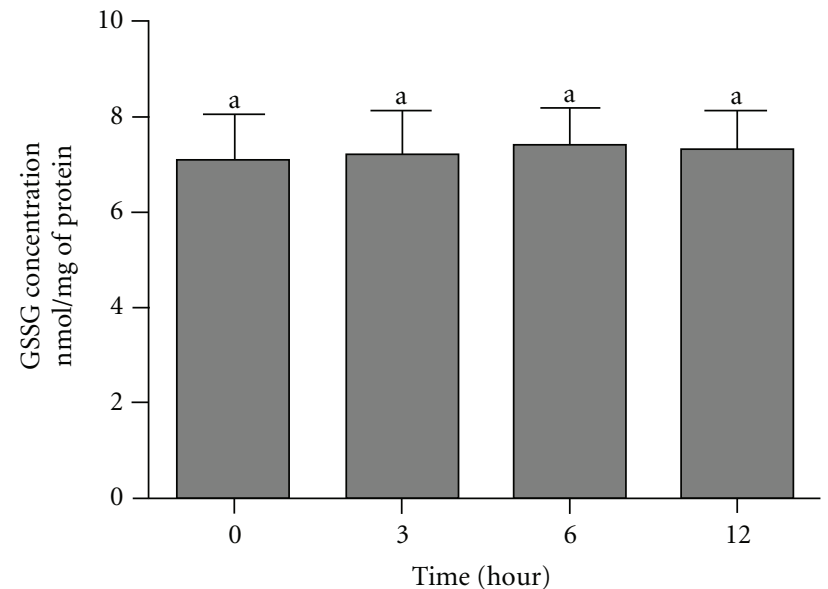

(b)

FIGURE 6: Measurement of intracellular GSH and GSSG in control and alantolactone-treated HepG2 cells for various time points. Cells were cultured in 6 well plates and treated with or without $40 \mu \mathrm{M}$ alantolactone for 3, 6, and $12 \mathrm{~h}$. Intracellular GSH and GSSG were measured according to the instructions of kit. Data are expressed as mean $\pm \mathrm{SD}(n=3)$. Columns not sharing the same superscript letter differ significantly $(P<0.0)$. 


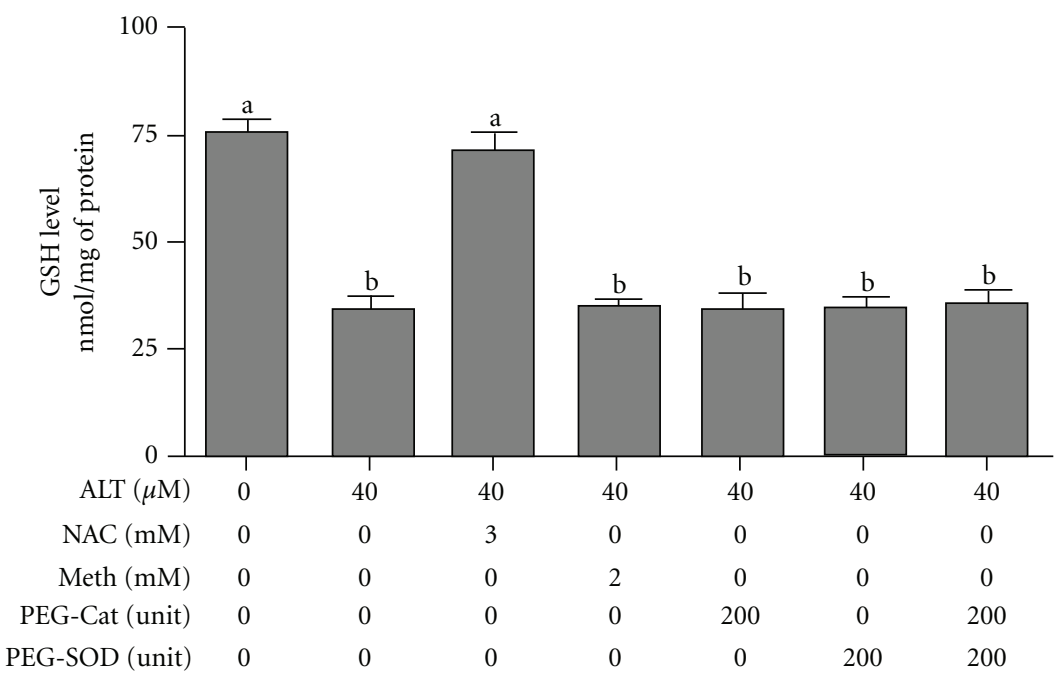

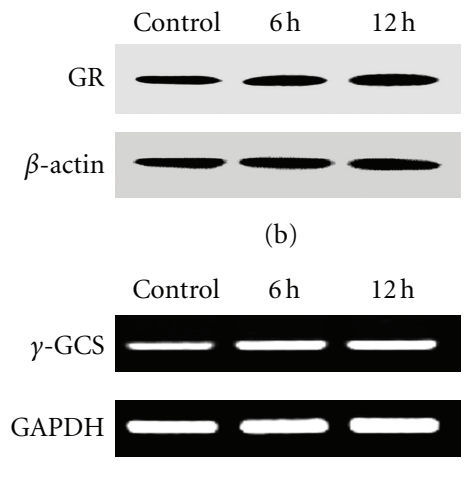

(c) (a)

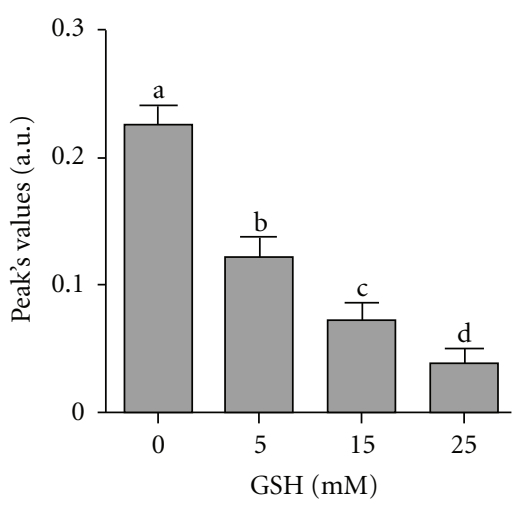

(d)

FIGURE 7: Measurement of GSH in the presence of various inhibitors and expression of genes involved in GSH metabolism. (a) HepG2 cells were treated with $40 \mu \mathrm{M}$ alantolactone for $6 \mathrm{~h}$ in the presence or absence of various inhibitors and concentration of GSH was measured according to kit instructions. Data are expressed as mean $\pm \mathrm{SD}(n=3)$. Columns not sharing the same superscript letter differ significantly $(P<0.05)$. (b) Cells were treated with $40 \mu \mathrm{M}$ alantolactone for indicated time points and cell lysates were subjected to Western blot analysis for the expression of glutathione reductase (GR). (c) Cells were treated with $40 \mu \mathrm{M}$ alantolactone for indicated time points and mRNA expression of $\gamma$-glutamyl cysteine synthetase ( $\gamma$-GCS) was determined by RT-PCR. (d) Alantolactone ( $1 \mathrm{mM})$ was incubated with indicated concentrations of GSH in medium for $30 \mathrm{~min}$ and amount of alantolactone was assessed using HPLC. The data shows that GSH decreased the amount of alantolactone in a dose-dependent manner. Data are expressed as mean $\pm \mathrm{SD}(n=3)$. Columns not sharing the same superscript letter differ significantly $(P<0.05)$.

was also observed. The data suggested that alantolactoneinduced cell death was through early apoptosis within $6 \mathrm{~h}$; however, with the increase of time, the late apoptotic rate also increased. Next, we treated the cells with $40 \mu \mathrm{M}$ alantolactone for $12 \mathrm{~h}$ in the presence of $3 \mathrm{mM} \mathrm{NAC}$ and the apoptosis rate was determined by flow cytometry. The data showed that NAC reversed the apoptotic effect of alantolactone indicating that alantolactone exerts an apoptotic effect in HepG2 cells through generation of ROS (Figure 3(e)).

3.4. Alantolactone Induces Increased Generation of ROS in HepG2 Cells. Intracellular ROS generation in HepG2 cells was measured by flow cytometry using DCFH-DA. The data demonstrated that the level of ROS in cells treated with $40 \mu \mathrm{M}$ alantolactone for 3,6 , and $12 \mathrm{~h}$ was $25 \%, 42 \%$, and $54 \%$, respectively (Figure 4). These values were significantly higher than those of the control group $(15 \%, P<0.05)$

3.5. Alantolactone Disrupts Mitochondrial Membrane Potential (MMP) in HepG2 Cells. Depolarization in MMP is a characteristic feature of apoptosis. Excessive intracellular ROS production has been shown to induce apoptosis by disrupting MMP $[19,20]$. To investigate the role of ROS in alantolactone-induced apoptosis, we determined MMP in HepG2 cells using Rhodamine 123 and flow cytometry. The data showed that alantolactone reduced the MMP in HepG2 cells in a time-dependent manner. As shown in Figure 5, MMP in cells treated with $40 \mu \mathrm{M}$ alantolactone for 3, 6, and $12 \mathrm{~h}$ was significantly lower (85\%, 79\%, and $62 \%$ versus $98 \%$ in the control group, $P<0.05)$. 
3.6. Alantolactone Reduces Intracellular GSH in HepG2 Cells. Intracellular GSH plays major roles in the maintenance of redox status and defense of oxidative stress. GSH depletion is an early hallmark observed in ROS mediated apoptosis. We therefore investigated the status of intracellular GSH in control and alantolactone-treated cells. A time-dependent study revealed that GSH depletion was significant from $3 \mathrm{~h}$ of treatment and increased over time (Figure 6(a)). Overproduction of ROS can oxidize GSH into GSSG. We, therefore, measured the level of GSSG in treated and untreated cells. As shown in Figure 6(b), no change in the level of GSSG in control and alantolactone-treated cells was found.

\subsection{Alantolactone Depletes Intracellular GSH via Direct Con-} jugation with GSH. The intracellular GSH depletion might be resulted either from an increased intracellular oxidation of GSH or a stimulated GSH extrusion through a specific carrier or inhibition of GSH synthesis. In addition, sesquiterpene lactones contain $\alpha$-methylene- $\beta$-lactone moiety which is highly reactive with cellular thiols and can deplete GSH by conjugating with sulfhydral group [21]. To shed light on the mechanism accounting alantolactone-mediated GSH depletion in HepG2 cells, we measured the concentration of GSH and GSSG in the culture medium. The concentration of GSH and GSSG in culture medium was not detectable. Overproduction of ROS can oxidize GSH into GSSG. Therefore, we incubated the cells with PEG-catalase and PEG-SOD and measured the level of GSH in cells. Pretreatment of cells with PEG-catalase and PEG-SOD alone or in combination did not prevent GSH depletion indicating that GSH depletion by alantolactone is not due to oxidation of GSH into GSSG (Figure 7(a)). During oxidative stress, GSH is oxidized to GSSG which is catalytically reduced back to GSH by glutathione reductase (GR). Next, we measured the expression of GR by Western blot analysis. The data showed that there was a slight increase in the expression level of GR in alantolactone-treated cell lysates, further confirming that GSH depletion is not linked with its oxidation to GSSG (Figure 7(b)).

Next, we asked if GSH depletion is resulted from GSH extrusion from cells. Therefore, we measured the level of GSH in cells treated with alantolactone for $6 \mathrm{~h}$ in the presence of $2 \mathrm{mM}$ methionine, a specific GSH carrier inhibitor. As shown in Figure 7(a), the inhibitor of GSH carrier (methionine) did not prevent GSH depletion, indicating that GSH depletion is not associated with GSH extrusion. To further support this finding, we measured GSH in a culture medium. The level of GSH in a culture medium was not detectable. The data demonstrate clearly that GSH depletion is not due to GSH extrusion.

Next, we treated the cells with $3 \mathrm{mM} \mathrm{NAC}$, a precursor molecule for GSH synthesis for $30 \mathrm{~min}$, followed by treatment with $40 \mu \mathrm{M}$ alantolactone for $6 \mathrm{~h}$, and intracellular GSH was determined in control and treated cells. As shown in Figure 6(a), pretreatment with NAC completely inhibited the depletion of intracellular GSH. To further exclude the possibility of GSH depletion by inhibition of GSH synthesis, we measured the mRNA expression of $\gamma$-glutamylcystein synthetase ( $\gamma$-GCS) which is a rate limiting enzyme in the synthesis of GSH. As shown in Figure 7(c), no change in the mRNA expression of $\gamma$-GCS was observed. The data demonstrate that GSH depletion is not associated with the inhibition of GSH synthesis. Therefore, the depletion of intracellular GSH by alantolactone is most probably the result of rapid binding of exomethylene moiety of alantolactone with intracellular GSH.

Finally, we incubated $1 \mathrm{mM}$ alantolactone with $0,5,15$, and $30 \mathrm{mM}$ GSH in DMEM medium without FBS at $37^{\circ} \mathrm{C}$ for $30 \mathrm{~min}$. Following incubation, the samples were analyzed by HPLC (Waters) using XTerra MS C18 $(5 \mu \mathrm{m}, 4.6 \times 150 \mathrm{~mm})$ column. The mobile phase was composed of acetonitrile (A) and water (B). The gradient program was as follows: 0-30 min, $A=65 \%$, and $B=35 \%$. The elution profile was analyzed at $227 \mathrm{~nm}$ by a UV detector. The data showed that the concentration of alantolactone decreased in the presence of increasing concentration of GSH (Figure 7(d)). The data demonstrate clearly the direct interaction of alantolactone with GSH. Taken together, the data demonstrate that alantolactone depletes intracellular GSH in HepG2 cells via direct conjugation with GSH.

\subsection{Alantolactone Inhibits STAT3 Activation and its Down-} stream Target Gene Bcl-2. Recent studies show that under oxidative stress, STAT3 is glutathionylated with a concomitant inhibition of its phosphorylation. In other words, GSH depletion inhibits STAT3 activation [16]. Because alantolactone reduced the intracellular GSH in HepG2 cells, we were interested if alantolactone could inhibit STAT3 activation. Thus we measured the expression of $\mathrm{pTyr}^{705}$ STAT3 in cells. As shown in Figure 8, alantolactone treatment decreased the expression of $\mathrm{pTyr}^{705}$ STAT3 in a time-dependent manner. After tyrosine phosphorylation ( $\mathrm{Tyr}^{705}$ ) of STAT3, it translocates into the nucleus where it increases the expression of genes implicated in cell proliferation such as $\mathrm{Bcl}-2$ and cyclin D1. Disruption of STAT3 signalling decreases the expression of antiapoptotic proteins and induces apoptosis in tumor cells. Therefore, we measured the expression of Bcl-2 family proteins using Western blot analysis. As expected, alantolactone treatment reduced the expression of antiapoptotic protein $\mathrm{Bcl}-2$ and increased the expression of proapoptotic protein Bax in a time-dependent manner (Figure 8).

3.9. Alantolactone Induces Caspase-3 Activation in HepG2 Cells. Mitochondrial-dependent apoptosis is initiated by recruitment and activation of caspases. Thus we analyzed whether caspase-3 was activated during alantolactoneinduced apoptosis of HepG2 cells. As shown in Figure 8, alantolactone stimulated the cleavage of caspase- 3 in a timedependent manner as demonstrated by the appearance of $32 \mathrm{kDa}$ and $17 \mathrm{kDa}$ fragments.

\section{Discussion}

Currently available chemotherapy remains ineffective to cure hepatoma mainly because of its high hepatotoxicity. Therefore, alternative therapeutic agents that kill cancer cells 
without or with low hepatotoxicity are highly desirable. Alantolactone, a sesquiterpene lactone, has been reported to possess antibacterial, antifungal, antihelminthic, and anticancer activities. In our previous report, we showed that alantolactone-induced apoptosis in U87 glioblastoma cells via GSH depletion without inducing toxicity in mouse liver and kidneys [15]. However, the molecular mechanism of GSH depletion by alantolactone remained unexplored. The present study was therefore conducted to evaluate whether alantolactone can deplete GSH and induce apoptosis in liver cancer cells. To study the anticancer activity of alantolactone towards liver cancer cells, we used HepG2 cell line as a model cell line. We found that alantolactone inhibited the growth and induced apoptosis in HepG2 cells in a dose- and timedependent manner as evident by Annexin $\mathrm{V}$ positive staining and caspase- 3 activation. This alantolactone-induced cell death was completely inhibited when cells were pretreated with NAC, a GSH precursor molecule. The data suggest that GSH depletion might be involved in apoptotic cell death induced by alantolactone. We therefore measured the level of intracellular GSH in control and treated cells. In accordance with our previous study [15], alantolactone reduced the level of GSH in HepG2 cells in a time-dependent manner.

It is well established that intracellular redox status or oxidative stress plays an important role in cancer cells apoptosis [21]. GSH is one of the most abundant intracellular antioxidants involved in the protection of cells against oxidative damage $[15,22]$. Depletion of intracellular GSH is an early hallmark in the onset of apoptosis [21, 23]. The intracellular GSH depletion might be resulted either from increased intracellular oxidation of GSH or stimulated GSH extrusion through a specific carrier or the inhibition of GSH synthesis or the direct conjugation of GSH with drug [21]. In the present study, alantolactone-mediated GSH depletion is unlikely to be due to the oxidation of GSH into GSSG since expression of GR is not inhibited and the level of GSSG remained unchanged in cells before and after treatment. Furthermore, GSH and GSSG were not detectable in culture medium and methionine (inhibitor of GSH carrier) did not prevent GSH depletion which excludes the possibility of GSH extrusion as the main mechanism for GSH depletion. The pretreatment of cells with NAC completely inhibited GSH depletion and mRNA expression of $\gamma$-GCS remained unchanged in control and alantolactone-treated cells. These sets of data demonstrate clearly that alantolactone-induced GSH depletion in HepG2 cells through a process that does not involve inhibition of GSH synthesis. Therefore, the depletion of intracellular GSH by alantolactone is most probably the result of direct conjugation of alantolactone with GSH. Alantolactone like other sesquiterpene lactones such as parthenolide and helenalin contains $\alpha$-methylene- $\gamma$-lactone moiety which can interact with sulfhydral group of GSH by means of Michael-type conjugation [21]. In order to ascertain the GSH depletion by direct conjugation with alantolactone, we incubated the alantolactone with GSH as described in Section 2 and samples were analyzed by HPLC. The data indicated that the amount of alantolactone decreased with the increasing concentration of GSH demonstrating their direct conjugation.

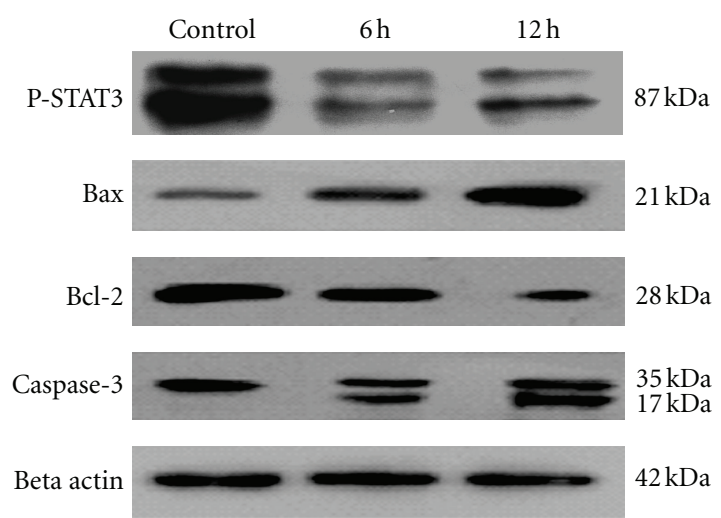

Figure 8: Effect of alantolactone on the expressions of apoptosis regulators. HepG2 cells were treated with or without $40 \mu \mathrm{M}$ alantolactone for 6 and $12 \mathrm{~h}$. The expression of Phospho-STAT3, Bax, $\mathrm{Bcl}-2$, and caspase- 3 was measured by Western blot analysis.

An increasing body of literature evidence underlines the critical role of redox reactions in the regulation of various cell functions. Reactive oxygen species are produced inside the cells during normal physiological processes of the cells which are being neutralized by the antioxidant system of the cells $[24,25]$. A precise balance between ROS production and antioxidant system's ability to scavenge ROS is critical for normal cellular functions. GSH is one of the most important intracellular antioxidant which plays the major role in protection of cells against oxidative damage. Depletion of GSH results in oxidative stress which is a known inducer of the transcription of specific genes involved in apoptosis [26]. In order to ascertain the mechanism by which alantolactoneinduced GSH depletion induces apoptosis in HepG2 cells, we measured the expression of pTyr $^{705}$ STAT3 and its downstream target protein $\mathrm{Bcl}-2$. We found that alantolactone treatment reduced the expression of phosphorylated STAT3 and Bcl-2 in a time-dependent manner. The inhibition of STAT3 by alantolactone is further supported by another recent study that parthenolide a sesquiterpene lactone that depletes GSH in HepG2 cells by direct conjugation with GSH also inhibits STAT3 expression [27]. Both alantolactone and parthenolide share a common $\alpha$-methylene- $\gamma$-lactone moiety which directly conjugates with the self-hydral group of GSH. However, the molecular mechanism by which GSH depletion inhibits STAT3 expression remained unexplored in the present study. Further study is needed to bridge the information link between GSH depletion and STAT3 inhibition.

GSH depletion and ROS generation (oxidative stress) are known to act as second messengers to activate diverse redox-sensitive signalling cascades including mitochondrial intrinsic apoptotic cascade through interaction with $\mathrm{Bcl}$ 2 family proteins $[20,28]$. Bcl-2 family proteins include a wide variety of antiapoptotic proteins such as Bcl-2 and proapoptotic proteins such as Bax, which are key players of mitochondrial outer membrane permeabilization and apoptosis regulation [20, 29]. GSH depletion and oxidative stress have been reported to activate and translocate 
proapoptotic protein Bax to outer mitochondrial membrane (OMM) where it forms oligomers which are thought to be important in the formation of permeability transition pores (PTP) and cytochrome c release [20, 22, 30]. Apart from Bax activation, ROS have also been shown to inhibit antiapoptotic protein Bcl-2 [20,30]. In the present study, the expression of Bax increased while the expression of $\mathrm{Bcl}-2$ decreased in alantolactone-treated cells in a time-dependent manner. The data demonstrated that alantolactone induced apoptosis in HepG2 cells through mitochondrial pathway. This mitochondrial apoptotic pathway was further confirmed by measuring the mitochondrial membrane potential (MMP) using flow cytometry. A significant reduction in MMP has been observed in alantolactone-treated cells, suggesting the opening of mitochondrial permeability transition pore. Therefore, we concluded that alantolactone can promote the opening of mitochondrial permeability transition pore by increasing the $\mathrm{Bax} / \mathrm{Bcl}-2$ ratio.

\section{Conclusion}

In conclusion our data provide evidence for the first time that alantolactone depletes GSH in HepG2 cells by direct conjugation with GSH. The GSH depletion resulted in the inhibition of phosphorylated STAT3 expression and oxidative stress. Consequently, inhibition of STAT3 activation and oxidative stress induced apoptosis in HepG2 cells by modulating mitochondrial Bcl-2 family proteins. Our results suggest that alantolactone may be a promising chemotherapeutic drug candidate for the treatment of liver cancer. Further investigation is needed to validate the contribution of alantolactone to tumor therapy in vivo.

\section{Conflict of Interests}

The authors have declared that they have no conflict of interests.

\section{Acknowledgments}

This work is supported by the Ministry of Education of Pakistan and the Chinese Scholarship Council of China.

\section{References}

[1] R. J. Thoppil and A. Bishayee, "Terpenoids as potential chemopreventive and therapeutic agents in liver cancer," World Journal of Hepatology, vol. 3, pp. 228-249, 2011.

[2] X. W. Tan, H. Xia, J. H. Xu, and J. G. Cao, "Induction of apoptosis in human liver carcinoma HepG2 cell line by 5-allyl-7-gendifluoromethylenechrysin," World Journal of Gastroenterology, vol. 15, no. 18, pp. 2234-2239, 2009.

[3] L. Andreana, G. Isgro, L. Marelli et al., "Treatment of hepatocellular carcinoma (HCC) by intra-arterial infusion of radioemitter compounds: trans-arterial radio-embolisation of HCC," Cancer Treatment Reviews, vol. 38, pp. 641-649, 2012.

[4] A. Alisi and C. Balsano, "Enhancing the efficacy of hepatocellular carcinoma chemotherapeutics with natural anticancer agents," Nutrition Reviews, vol. 65, no. 12, pp. 550-553, 2007.
[5] T. Beppu, K. Sugimoto, K. Shiraki et al., "Clinical significance of tumor markers in detection of recurrent hepatocellular carcinoma after radiofrequency ablation," International Journal of Molecular Medicine, vol. 26, no. 3, pp. 425-433, 2010.

[6] P. J. Johnson, "Non-surgical treatment of hepatocellular carcinoma," $H P B$, vol. 7, no. 1, pp. 50-55, 2005.

[7] B. Liu, G. Wang, J. Yang, X. Pan, Z. Yang, and L. Zang, "Berberine inhibits human hepatoma cell invasion without cytotoxicity in healthy Hepatocytes," PLoS One, vol. 6, no. 6, Article ID e21416, 2011.

[8] T. W. Chung, Y. C. Lee, and C. H. Kim, "Hepatitis B viral HBx induces matrix metalloproteinase- 9 gene expression through activation of ERK and PI-3K/AKT pathways: involvement of invasive potential," The FASEB Journal, vol. 18, no. 10, pp. 1123-1125, 2004.

[9] W. Y. Wu, J. Li, Z. S. Wu, C. L. Zhang, and X. L. Meng, "STAT3 activation in monocytes accelerates liver cancer progression," BMC Cancer, vol. 11, article 506, 2011.

[10] L. Lin, S. Deangelis, E. Foust et al., "A novel small molecule inhibits STAT3 phosphorylation and DNA binding activity and exhibits potent growth suppressive activity in human cancer cells," Molecular Cancer, vol. 9, article 217, 2010.

[11] A. Ghantous, H. Gali-Muhtasib, H. Vuorela, N. A. Saliba, and N. Darwiche, "What made sesquiterpene lactones reach cancer clinical trials?” Drug Discovery Today, vol. 15, no. 15-16, pp. 668-678, 2010.

[12] S. Zhang, Y. K. Won, C. N. Ong, and H. M. Shen, "Anti-cancer potential of sesquiterpene lactones: bioactivity and molecular mechanisms," Current Medicinal Chemistry, vol. 5, no. 3, pp. 239-249, 2005.

[13] H. C. Pal, I. Sehar, S. Bhushan, B. D. Gupta, and A. K. Saxena, "Activation of caspases and poly (ADP-ribose) polymerase cleavage to induce apoptosis in leukemia HL-60 cells by Inula racemosa," Toxicology in Vitro, vol. 24, no. 6, pp. 1599-1609, 2010.

[14] A. Trendafilova, C. Chanev, and M. Todorova, "Ultrasoundassisted extraction of alantolactone and isoalantolactone from Inula helenium roots," Pharmacognosy Magazine, vol. 6, no. 23, pp. 234-237, 2010.

[15] M. Khan, F. Yi, A. Rasul et al., "Alantolactone induces apoptosis in glioblastoma cells via GSH depletion, ROS generation, and mitochondrial dysfunction," IUBMB Life, vol. 64, no. 9, pp. 783-794, 2012.

[16] E. Butturini, E. Cavalieri, A. C. de Prati et al., "Two naturally occurring terpenes, dehydrocostuslactone and costunolide, decrease intracellular GSH content and inhibit STAT3 activation," PLoS One, vol. 6, no. 5, Article ID e20174, 2011.

[17] M. Khan, B. Zheng, F. Yi et al., "Pseudolaric acid B induces caspase-dependent and caspase-independent apoptosis in U87 glioblastoma cells," Evidence-Based Complementary and Alternative Medicine, vol. 2012, Article ID 957568, 11 pages, 2012.

[18] M. Khan, B. Yu, A. Rasul et al., "Jaceosidin induces apoptosis in U87 glioblastoma cells through G2/M phase arrest," EvidenceBased Complementary and Alternative Medicine, vol. 2012, Article ID 703034, 12 pages, 2012.

[19] X. Ling, Y. Zhou, S. W. Li, B. Yan, and L. Wen, "Modulation of mitochondrial permeability transition pore affects multidrug resistance in human hepatocellular carcinoma cells," International Journal of Biological Sciences, vol. 6, no. 7, pp. 773-783, 2010.

[20] Y. B. Ji Yu-Bin, Z. Y. Qu, and X. Zou, "Juglone-induced apoptosis in human gastric cancer SGC-7901 cells via the mitochondrial 
pathway," Experimental and Toxicologic Pathology, vol. 63, no. 1-2, pp. 69-78, 2011.

[21] S. Zhang, C. N. Ong, and H. M. Shen, "Critical roles of intracellular thiols and calcium in parthenolide-induced apoptosis in human colorectal cancer cells," Cancer Letters, vol. 208, no. 2, pp. 143-153, 2004.

[22] P. Guha, A. Dey, R. Sen, M. Chatterjee, S. Chattopadhyay, and S. K. Bandyopadhyay, "Intracellular GSH depletion triggered mitochondrial bax translocation to accomplish resveratrolinduced apoptosis in the U937 cell line," Journal of Pharmacology and Experimental Therapeutics, vol. 336, no. 1, pp. 206-214, 2011.

[23] M. L. Circu and T. Y. Aw, "Glutathione and apoptosis," Free Radical Research, vol. 42, no. 8, pp. 689-706, 2008.

[24] S. Deng, Y. Yang, Y. Han et al., "UCP2 inhibits ROS-mediated apoptosis in A549 under hypoxic conditions," PLoS One, vol. 7, Article ID e30714, 2012.

[25] V. Sharma, C. Joseph, S. Ghosh, A. Agarwal, M. K. Mishra, and E. Sen, "Kaempferol induces apoptosis in glioblastoma cells through oxidative stress," Molecular Cancer Therapeutics, vol. 6, no. 9, pp. 2544-2553, 2007.

[26] C. Kretz-Remy and A. P. Arrigo, "Gene expression and thiol redox state," Methods in Enzymology, vol. 348, pp. 200-215, 2002.

[27] D. Carlisi, A. D’Anneo, L. Angileri et al., "Parthenolide sensitizes hepatocellular carcinoma cells to trail by inducing the expression of death receptors through inhibition of STAT3 activation," Journal of Cellular Physiology, vol. 226, no. 6, pp. 1632-1641, 2011.

[28] Y. Tsujimoto and S. Shimizu, "Role of the mitochondrial membrane permeability transition in cell death," Apoptosis, vol. 12, no. 5, pp. 835-840, 2007.

[29] M. L. Circu and T. Y. Aw, "Reactive oxygen species, cellular redox systems, and apoptosis," Free Radical Biology and Medicine, vol. 48, no. 6, pp. 749-762, 2010.

[30] E. Mayola, C. Gallerne, D. D. Esposti et al., "Withaferin A induces apoptosis in human melanoma cells through generation of reactive oxygen species and down-regulation of Bcl-2," Apoptosis, vol. 16, pp. 1014-1027, 2011. 

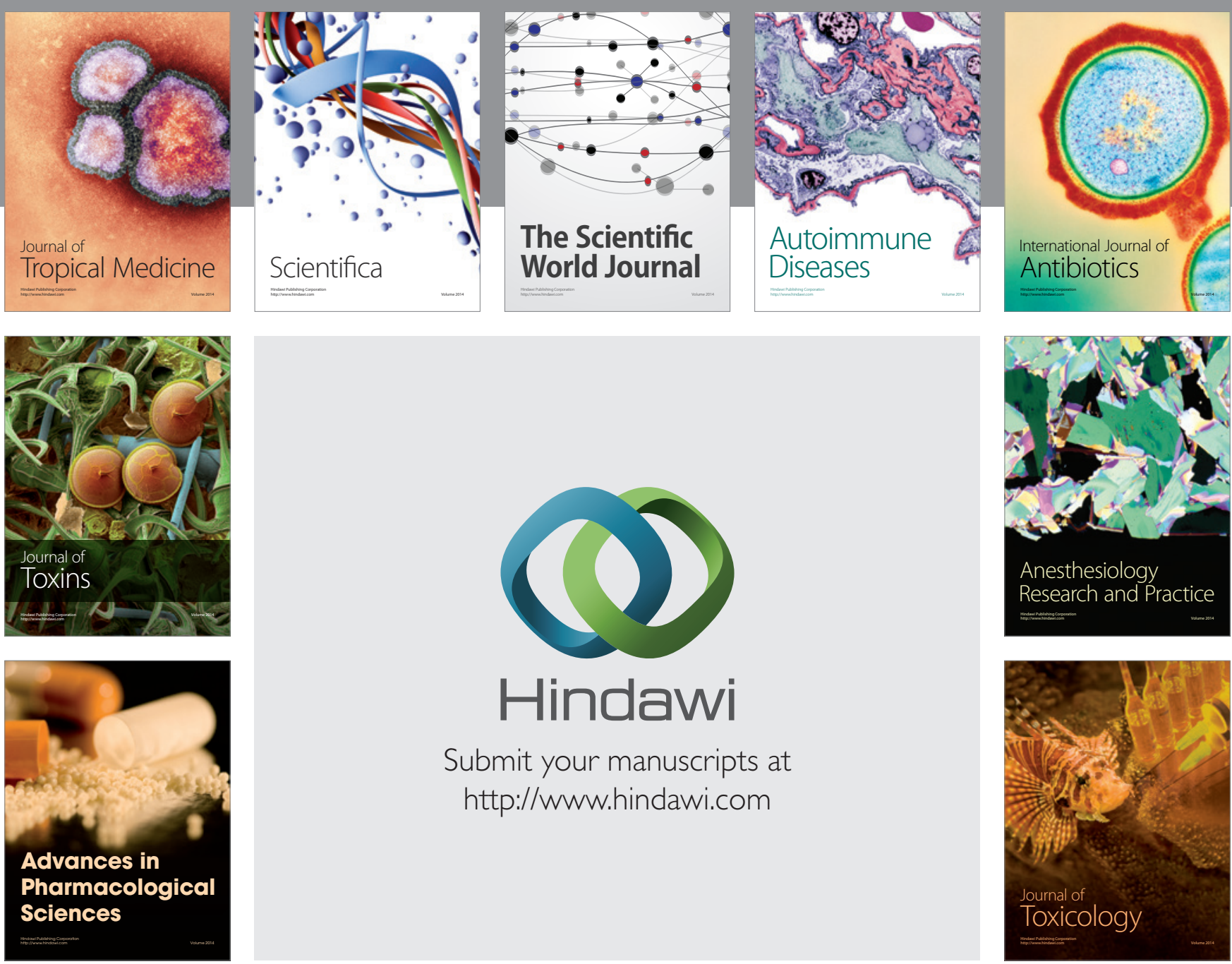

\section{Hindawi}

Submit your manuscripts at

http://www.hindawi.com
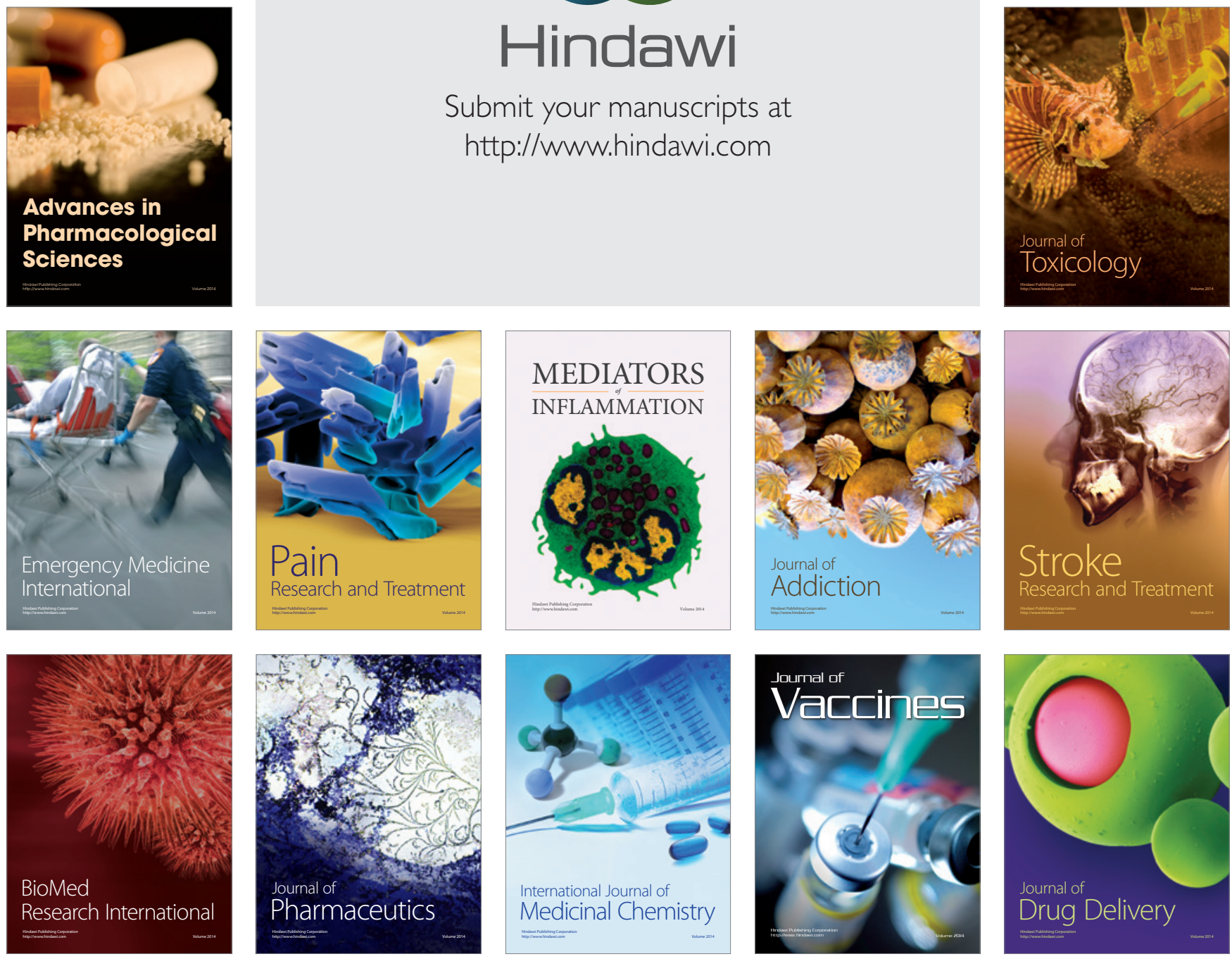